\title{
Root morphology in response to nitrogen supply in mid-season indica rice cultivars released in different decades
}

\author{
Yikai Zhang ${ }^{1}$, Huizhe Chen ${ }^{1}$, Yuping Zhang ${ }^{1}$, Jing Xiang ${ }^{1}$, Guangmei Ji ${ }^{1,2} \&$ Defeng Zhu ${ }^{1 *}$ \\ ${ }^{1}$ State Key Laboratory of Rice Biology, China National Rice Research Institute, Hangzhou 310006, China; \\ ${ }^{2}$ Rice Research Institute of Guizhou Province, Guiyang 550006, China
}

Received September 18,2016; accepted October 21,2016; published online December 1,2016

Citation: Zhang, Y., Chen, H., Zhang, Y., Xiang, J., Ji, G., and Zhu, D. (2016). Root morphology in response to nitrogen supply in mid-season indica rice cultivars released in different decades. Sci China Life Sci 60, 439-442. doi: 10.1007/s11427-016-0326-7

Dear Editor,

Roots, as a major organ of plants, are involved in nutrient and water acquisition, and might play a vital role in yield increase and efficient $\mathrm{N}$ absorption with genetic improvement. Because of the great differences in growth period and pattern between the old and new rice cultivars, it is difficult to clarify how genetic improvements contribute to root growth in rice. For example, for a solution culture system, $\mathrm{Wu}$ et al. suggested that total root length increased with increasing year of release for maize hybrids, while shoot dry weight, root dry weight and leaf area decreased (Wu et al., 2011). However, using similar materials to $\mathrm{Wu}$ et al. in the field, Chen et al. reported that dry matter production had increased, and modern maize since 1990 had reduced root length density (Chen et al., 2014). Thus, they did not rule out that the growth period and shoot growth potential of cultivars may be an important factor (Peng et al., 2010). So, in the present study, the effect of modern plant breeding on changes in rice root traits was investigated under equivalent $\mathrm{N}$ absorption of shoots by multiple sampling.

The experiments were conducted at the experimental base of the China National Rice Research Institute in Fuyang, China. Eleven representative mid-season indica rice (Oryza sativa L.) cultivars applied in production in the Mid-

*Corresponding author (email: cnrice@qq.com)
dle-Lower Yangtze Area during the last 60 years were used to investigate root responses to variations in $\mathrm{N}$ availability using a solution culture system (Table S1 in Supporting Information). The experiment aimed to evaluate whether the high $\mathrm{N}$ inputs used in modern rice breeding programs affect root growth. Seedlings were supplied with either 0.29 (low $\mathrm{N}, \mathrm{LN}$ ) or $2.9 \mathrm{mmol} \mathrm{L}^{-1} \mathrm{~N}$ (high $\mathrm{N}, \mathrm{HN}$ ). Plants were sampled on the first day of $\mathrm{N}$ treatments and second on five weeks, other sampled every two days, making five times in all. Then, one of the near $\mathrm{N}$ absorption was selected, and the root traits of all cultivars were compared under the equivalent $\mathrm{N}$ absorption of plant (Table $\mathrm{S} 2$ in Supporting Information).

The results showed that biomass of rice seedlings significantly changed with increasing year of cultivar release under equivalent amounts of $\mathrm{N}$ absorption per plant (Figure 1A, Table S3 in Supporting Information). The new rice cultivars had larger shoot dry weight compared with old cultivars for HN ( $r=0.7977)$. Correspondingly, here, we found that root dry weight consists of thick adventitious roots and was higher in newly developed cultivars under high-N treatment (Figure 1B). The growth of roots is closely related to the supply of photosynthate from the shoot. Zhang et al. observed that new super rice cultivars had a larger leaf area index than conventional cultivars throughout their growth period (Zhang et al., 2009). Under equivalent $\mathrm{N}$ absorption, new cultivars supplied more carbohydrates to roots and maintained their vigorous function. This suggests that the larger 

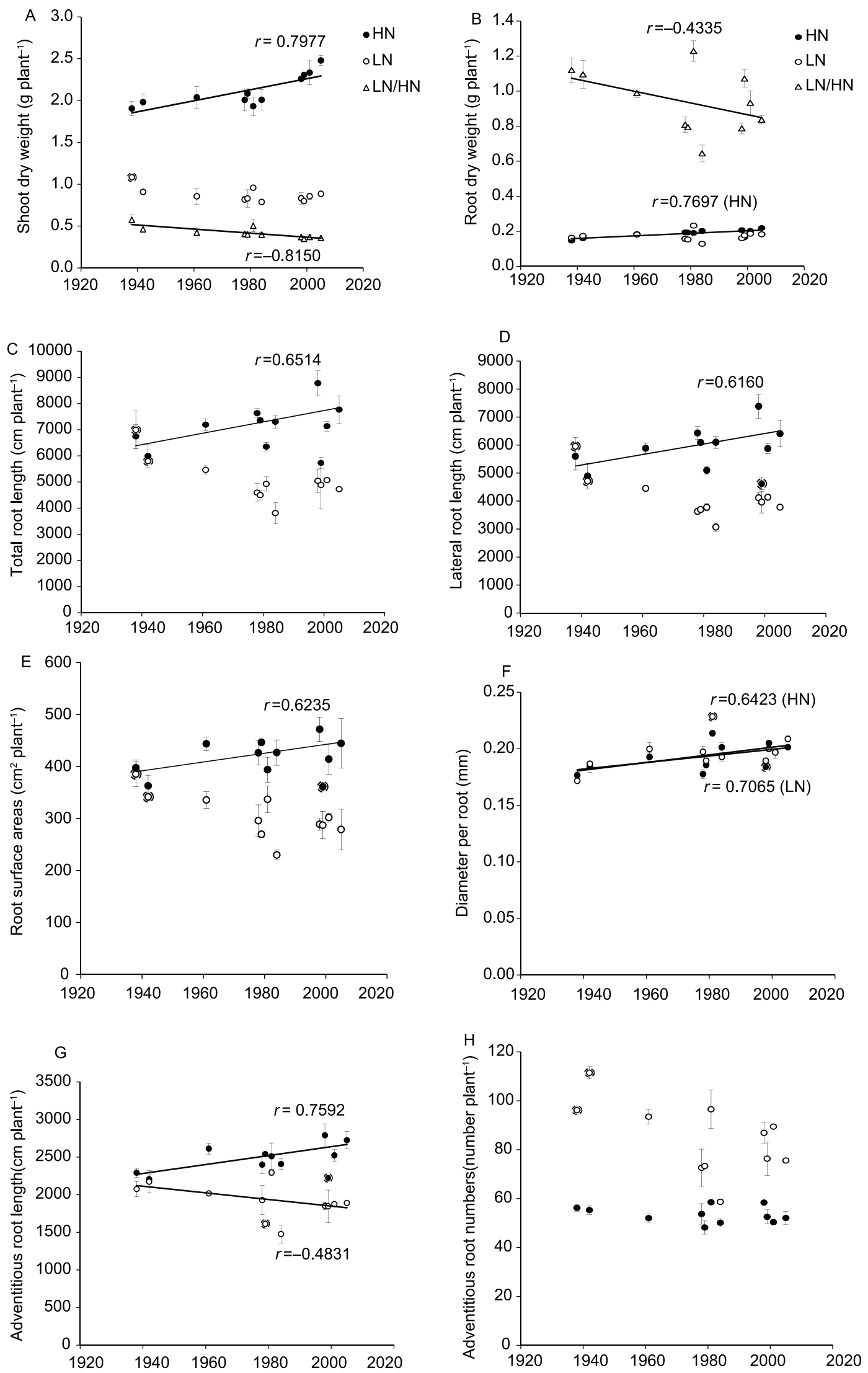

Figure 1 Changes in shoot dry weight (A), root dry weight (B), total root length (C), lateral root length (D), root surface areas (E), diameter per root (F), adventitious root length $(\mathrm{G})$ and adventitious root number $(\mathrm{H})$ during the genetic improvement of mid-season indica rice cultivars under equivalent $\mathrm{N}$ absorption. $\circ$, low $\mathrm{N}$ treatment; $\bullet$, high $\mathrm{N}$ treatment. Data point omitted from regression analysis is circled. Each value is the mean \pm standard error of three replicates. 
biomass contributes to higher grain yield in new ice cultivars.

Root morphology markedly changed with increasing year of cultivar release for equivalent amounts of $\mathrm{N}$ absorption, and showed a strong interaction between the factors of $\mathrm{N}$ and genotype (Figure 1). In contrast to the greater root dry weight, new cultivars showed the same trends on the total root and lateral root lengths under equivalent amounts of $\mathrm{N}$ absorption (Figure 1C and D), implying thicker roots in modern cultivars. This is beneficial for the new cultivars to support their larger shoots, and to be more resistant to root lodging at high plant densities. Lateral roots constituted the majority of the total root length, and their spatial distribution and density in soil determines the acquisition of nutrients. Since N content is the same under both LN and HN, N utilization efficiency is increased under both $\mathrm{N}$ levels. The increase in root length under $\mathrm{HN}$ may have a better effect for $\mathrm{N}$ acquisition. The new cultivars had more tillers under HN conditions (Figure S1 in Supporting Information), which contributed to the larger root systems. Additionally, the newly developed cultivars had greater root diameter under HN (Figure 1F). Adventitious root length increased with more recently developed cultivars under HN. A longer adventitious root can increase vertical or horizontal root distribution and therefore explore a larger soil volume. Gowda et al. showed the new cultivars were more effective at extracting water at greater depths under conditions of water limitation (Gowda et al., 2011). The distribution of adventitious roots determines root architecture and their elongation is essential for $\mathrm{N}$ and water uptake in deep soil. The only exception among newer cultivars was Liangyoupeijiu, which had lower total (Figure 1C), lateral (Figure 1D) and adventitious root lengths (Figure 1G). Fewer roots of Liangyoupeijiu would reduce the carbon cost of root growth and competition for nutrient resources, but would be disadvantageous by having less lodging resistance.

Although root dry weight, total root length and lateral root length per plant were higher in newly developed cultivars for $\mathrm{HN}$, they were similar to those of older cultivars except for early tall cultivars Shenglixian and Zhongnong 4 for LN treatment under equivalent $\mathrm{N}$ absorption. The decreasing LN/HN ratio ( $r=-0.4334)$ also suggest that roots of the new super rice cultivars were less responsive to LN (Figure 1A). Modern rice cultivars are commonly selected under high nutrient supply, resulting in their high tolerance to high $\mathrm{N}$ rates and less response of root growth to low N (Wu et al., 2011). Adventitious root length showed no change in the cultivars prior to the 1980s, but was decrease in later cultivars for LN (Figure 1G).
The $\mathrm{R} / \mathrm{S}$ ratio and root diameter were also higher in newly developed cultivars for LN (Figure S2 in Supporting Information, Figure 1F). Some reports also showed that longer axial roots appeared to be more important for efficient $\mathrm{N}$ acquisition in maize. Under $\mathrm{N}$-deficient conditions, the improvement in adventitious root growth may contribute greatly to high stress tolerance in new cultivars.

This study demonstrated that root growth and its response in acquiring $\mathrm{N}$ for rice cultivars released during the last 60 years had changed markedly with equivalent $\mathrm{N}$ absorption per plant. New rice cultivars had greater root dry matter and total, lateral and adventitious root lengths than older cultivars, which corresponded to their larger shoot biomass at high $\mathrm{N}$ with equivalent $\mathrm{N}$ content per plant. The modern rice cultivars produced more dry matter and larger roots and contributed to high-yield formation under equivalent $\mathrm{N}$ absorption during sufficient $\mathrm{N}$ supply. However, the response of root growth and dry matter accumulation to low $\mathrm{N}$ stress was similar in both modern and older rice cultivars with equivalent $\mathrm{N}$ mg per plant. Thus, modern rice breeding has not specifically enhanced root responsiveness to $\mathrm{N}$ availability, and root growth should be considered as part of rice breeding programs in the future.

Compliance and ethics The author(s) declare that they have no conflict of interest.

Acknowledgements The authors thank the National Mid-term Genebank for Rice of China National Rice Research Institute for providing the rice germplasm collection. This work was supported by the Zhejiang Provincial Natural Science Foundation (LY16C130006), the Basic Research Foundation of National Commonweal Research Institute (2014RG004-3), and the National Natural Science Foundation (31501272).

Chen, X., Zhang, J., Chen, Y., Li, Q., Chen, F., Yuan, L., and Mi, G. (2014). Changes in root size and distribution in relation to nitrogen accumulation during maize breeding in China. Plant Soil 374, 121-130.

Gowda, V.R.P., Henry, A., Yamauchi, A., Shashidhar, H.E., and Serraj, R. (2011). Root biology and genetic improvement for drought avoidance in rice. Field Crops Res 122, 1-13.

Peng, Y., Niu, J., Peng, Z., Zhang, F., and Li, C. (2010). Shoot growth potential drives $\mathrm{N}$ uptake in maize plants and correlates with root growth in the soil. Field Crops Res 115, 85-93.

Wu, Q.P., Chen, F.J., Chen, Y.L., Yuan, L.X., Zhang, F.S., and Mi, G.H. (2011). Root growth in response to nitrogen supply in Chinese maize hybrids released between 1973 and 2009. Sci China Life Sci 54, 642-650.

Zhang, H., Xue, Y., Wang, Z., Yang, J., and Zhang, J. (2009). Morphological and physiological traits of roots and their relationships with shoot growth in "super" rice. Field Crops Res 113, 31-40.

Open Access This article is distributed under the terms of the Creative Commons Attribution License which permits any use, distribution, and reproduction in any medium, provided the original author(s) and source are credited. 


\section{SUPPORTING INFORMATION}

Table S1 The characteristics of 11 mid-season indica rice cultivars used in this experiment

Table S2 N content of mid-season indica rice cultivars in the shoot at harvest

Table S3 Variance analysis of effects of N, genotype, and N×genotype interaction on shoot and root growth parameters of rice

Figure S1 Changes in tiller numbers during the genetic improvement of mid-season indica rice cultivars under equivalent $\mathrm{N}$ absorption.

Figure S2 Changes in root to shoot biomass (R/S) during the genetic improvement of mid-season indica rice cultivars under equivalent $\mathrm{N}$ absorption.

The supporting information is available online at life.scichina.com and www.springerlink.com. The supporting materials are published as submitted, without typesetting or editing. The responsibility for scientific accuracy and content remains entirely with the authors. 